\title{
Individuación del individuo: Una posibilidad de resignificar las relaciones de intimidad
}

\author{
Karina Arias Díaz* \\ Natalia Hernández Mary**
}

\begin{abstract}
RESUMEN
El presente artículo tiene como objetivo develar la resignificación de los espacios de intimidad de las relaciones interpersonales en el contexto de las sociedades modernas, en las cuales se ha desarrollado un proceso clave: la individuación del individuo. Desde las propuestas que ofrece Luhman surge la posibilidad de afirmar que los espacios de intimidad existen y son validados por los individuos modernos.
\end{abstract}

Palabras clave: Teoría de sistema - individualidad - individuación relaciones interpersonales - intimidad

\section{Individuación do indivíduo: uma possibilidade de resignificar as relações de intimidai}

\begin{abstract}
RESUMO
O presente artigo tem como objectivo develar a resignificación dos espaços de intimidai das relações interpersonales no contexto das sociedades modernas, nas quais se desenvolveu um processo finque: a individuación do indivíduo. Desde as propostas que oferece Luhman surge a possibilidade de afirmar, que os espaços de intimidai existem e são validados pelos indivíduos modernos.
\end{abstract}

Palavras-chave: Teoria de sistema - individualidad - individuación relações interpersonales - intimidai

* Chilena. Trabajadora Social. Directora Centro de Encuentro del Adulto Mayor, CEAM La Pintana, Programa de Atención Domiciliaria al Adulto Mayor PADAM La Pintana y Condominio de Adultos Mayores Padre Hurtado de La Granja. Fundación Hogar de Cristo. Correo electrónico: karias@uc.cl

** Chilena. Trabajadora Social. Docente Departamento de Trabajo Social Universidad Alberto Hurtado. Coordinadora Operativa Programa Acompañamiento Solidario (PAS), Gesta, Fundación Marista Por la Solidaridad. Correo electrónico: nataliahernandez@fundaciongesta.cl 


\title{
Individuation of the individual meanings to the possibility of intimate relationships
}

\begin{abstract}
The present test(essay) takes as an aim(lens) develar the resignificance(remeaning) of the spaces of intimacy of the interpersonal relations in the context of the modern societies, in which a key process has developed: the individuation of the individual. From the offers that Luhman offers there arises the possibility of affirming, that the spaces of intimacy exist and are validados for the modern individuals.
\end{abstract}

Key words: System theory - individuality - individuation - interpersonal relations - intimidate 


\section{Sociedad moderna e individuación del individuo}

La sociedad, según Luhmann (1998), es un sistema autopoiético de comunicaciones, es un sistema autorreferente que evoluciona y tiene en la comunicación su rasgo característico. No está compuesta de hombres, sino de comunicaciones y está constituida por diversos sistemas parciales: jurídico, económico, político, religioso, entre otros. A su vez, cada uno de ellos, son sistemas autopoiéticos que tienen a los otros sistemas parciales como entorno (Mascareño, 2006)

En la sociedad moderna estos subsistemas se han configurado de manera particular, generando altos niveles de diferenciación y formas de especificación funcional que han especializado las respuestas comunicativas de su entorno. Según Luhmann (1998), esto responde a la diferenciación de los sistemas sociales que refiere al incremento de la selectividad a través de una división interna y recurrente de la sociedad, aumentando las posibilidades disponibles para la variación o la elección.

Giddens (1995) señala que el rasgo específico de la modernidad es la distinción tiempo-espacio "las vinculaciones específicas entre espacio y tiempo se relajarían, se harían contingentes, pasando a descansar, pues, sólo en convenciones y a través del (...) entrelazamiento recursivo de las determinaciones de la acción con otras acciones o posibilidades de acción, (...) esta transformación repercutiría de manera "globalizante" sobre la totalidad de los dominios de acción. Las circunstancias locales determinarían cada vez menos el modo de vida y las consecuencias afectarían a estructuras y semánticas" (Giddens 1998: 135)

De acuerdo a lo anterior, el proceso de globalización -entendido como el aumento del volumen e intensidad del tráfico, la comunicación y los intercambios- que sobrepasa las fronteras, ha generado el incremento de la especialización y diferenciación de los sistemas en la sociedad moderna. A su vez, ha primado una constante y creciente autonomía y racionalización en los sistemas. Autonomía en términos de regirse sólo por sus propias y exclusivas certezas; y racionalización como proceso de organización de los sistemas autónomos, de acuerdo con relaciones cada vez más eficientes entre fines y medios, donde los fines a considerar son únicamente 
los propios (PNUD, 1994), potenciándose así la clausura operativa y el acoplamiento estructural de los sistemas.

En otras palabras, los sistemas diferenciados se relacionan entre sí en términos de coordinación funcional. Cada uno toma en cuenta los otros sistemas en cuanto a entornos de recursos y obstáculos que pueden ser aprovechados o evitados en función de los propios fines. La coordinación funcional puede ser más o menos espontánea o puede asumir la forma de regulaciones e instituciones (PNUD, 1998).

Entre los efectos ocasionados por la globalización podemos mencionar el traspaso del poder o influencia de las comunidades locales y países al espacio mundial, las transacciones económicas tienen un alcance nunca antes visto, el nivel del comercio mundial abarca un espectro más amplio de bienes y servicios, surgen cambios en el sistema de comunicación y se crean nuevas zonas económicas y culturales dentro y a través de países.

A nivel de los individuos la sociedad moderna potencia el desarrollo de oportunidades y de las tecnologías de información, además de ampliar las libertades para elegir, lo que conlleva al aumento de la capacidad para diseñar o escoger por sí mismos el tipo de vida que desean.

Sin embargo, la globalización no está evolucionando de manera equitativa, sus consecuencias no son del todo benignas. Giddens señala: "La vería destrozando culturas locales, ampliando las desigualdades sociales y empeorando la suerte de los marginados. La globalización (...) crea un mundo de ganadores y perdedores, unos pocos en el camino rápido hacia la prosperidad, la mayoría condenada a una vida de miseria y desesperación" (Giddens 2000: 27-28).

De tal modo que es un error pensar que la globalización sólo tiene relación con los "grandes sistemas", pues, como menciona Giddens "no tiene que ver sólo con lo que hay "ahí afuera", remoto y alejado del individuo. Es también un fenómeno de "aquí adentro", que influye en los aspectos íntimos y personales de nuestras vidas" (Giddens 2000: 24-25).

Así, la modernidad altera radicalmente la vida cotidiana y afecta a los aspectos más personales de la experiencia. "La modernidad se ha 
de entender en un plano institucional; pero los cambios provocados por las instituciones modernas se entretejen directamente con la vida individual y, por tanto, con el yo" (Giddens 1995: 9).

Con las libertades ${ }^{1}$ obtenidas, a través de la modernización, los individuos se vieron enfrentados al desafío de ir autoconstruyendo su propio ser. El desarrollo de instituciones de carácter más autónomo van marcando la soledad de la individualidad, lo que se traduce en una situación de poca aceptación y tolerancia por parte de los individuos, pues se ven enfrentados a múltiples posibilidades que constituyen a las sociedades modernas (Mascareño, 2006).

Así, en una sociedad ampliamente diferenciada, cuyos sistemas poseen clausura operativa -son capaces de autorreproducirse- y apertura cognitiva, y donde la comunicación entre sistemas se genera mediante un entrelazamiento recursivo de comunicaciones, seleccionando la información que les es atractiva de acuerdo a los propios códigos de cada sistema; el individuo debe ser capaz de adaptarse y desempeñar diversos tipos de "roles" de modo de ser "atractivo" a otros sistemas, permitiendo ser irritados y acoplarse estructuralmente. Esto implica que el individuo no se queda en un statu quo, sino más bien se encuentra en un constante movimiento, puesto que la realización de los roles le implica estar efectuando frecuentes elecciones. A lo anterior se agrega la permanente contingencia a la que el individuo está enfrentando, debiendo escoger continuamente, lo que se puede traducir en la selección del rol que desea desempeñar para afectar al sistema en cuestión.

Ahora bien, en relación al individuo, Luhmann afirma que éste ocupa un lugar en los sistemas autopoiéticos, autorreferenciales, donde las relaciones operan bajo mecanismos de selectividad. Selectividad que disminuye, pero a la vez aumenta la complejidad del mundo, el sujeto observado no se encuentra en el sistema, sino que en el entorno. Para Luhmann debe sustituirse la dualidad individuo/ sociedad por la de sistema/entorno, el sujeto es el resultado de una distinción, de una observación, para lo cual debe situarse en el entorno y no en el sistema (Avaria, 2001).

$1 \quad \mathrm{Al}$ referirnos a las libertades, se hace referencia a que en las sociedades modernas los individuos tienen la posibilidad de elegir el camino que desean para sí, y no los designios de una sociedad estratificada de otras épocas. 
El problema es que las orientaciones de la sociedad son cambiantes, contradictorias entre sí y los sistemas presentan exigencias difíciles de eludir, por lo que la construcción de un proyecto de vida satisfactorio constituye una fuente de tensión importante para los individuos. Para adentrarse en la temática del individuo, desde la perspectiva que brinda la teoría de sistemas, se requiere profundizar en los procesos de individuación.

La individuación se produce debido a la autoconfrontación -como proceso asistido por la modernidad- consigo mismo en medio de unidades específicas de socialización (los sistemas). La individualización sería una búsqueda escogida, donde es el individuo quien busca constituirse como ser único que toma sus propias decisiones, en cambio la individuación, es una búsqueda obligada por las características de las sociedades modernas (Robles, 1999).

La individuación significará varios fenómenos de acuerdo a lo que señala Robles (1999), en principio la sustitución de formas de vida, caracterizadas por la propia escenificación de la biografía, no es una opción que pueda ser escogida o desechada, es más bien una radicalización de la individualización, no existen posibles orientaciones para la construcción de estas biografías; la política estará más bien desplazada a la vida de los individuos, se produce una desactivación de la política, la individuación daría origen a identidades híbridas. La individuación sería entonces, una radicalización de la individualización.

Esta radicalización permite visualizar las opciones de los individuos, las cuales están dirigidas a acoplarse con los diferentes sistemas que constituyen el tejido social. El acoplarse con dichos sistemas implica que han debido optar por ciertas selecciones que le permitan una aventajada relación, de manera que pueda ser incluido en este sistema mayor. Esta condición ha provocado que el individuo en sí mismo haya perdido espacios de valorización propia, más bien se visibiliza y se aprecia aquellos aspectos que son útiles para su relación con los sistemas que se han mencionado.

El proceso de individuación, así, obliga al individuo a ser capaz de planificar a largo plazo, a adaptarse al cambio constante que produce la especialización de los sistemas en un entorno contingente, a organizarse a la vez que improvisar, a fijar metas, a reconocer los obstáculos, a aceptar las derrotas y a intentar nuevas salidas. El 
individuo se siente despojado y solo en un mundo donde carece de los apoyos psicológicos y del sentimiento de seguridad que le procuraban otros ambientes más tradicionales.

Asimismo, la radicalización de la individualización exige al individuo responder de determinadas formas ante las condiciones del mercado laboral, el sistema educativo, el Estado, entre otros sistemas. Estas sobreexigencias van excluyendo la posibilidad de los individuos de encontrar autorrealización en éstos ámbitos, pues el mercado visualiza al individuo en cuanto a trabajador; el sistema educacional en cuanto estudiante; el sistema político en cuanto ciudadano; pero, ¿qué sistema permite la valoración del individuo por sí mismo?

Es así como se cree que aún existen espacios donde se valora el propio individuo, por ser él mismo: los espacios, denominados como "íntimos", que se constituyen en las relaciones interpersonales de los individuos. La intimidad tiene su propia reflexividad y sus formas particulares de referencial interno. Dentro de este espacio nace la "relación pura" (Giddens, 1995), es decir, una relación en la que han desaparecido los criterios externos, existiendo la relación, sólo por las recompensas que puede proporcionar ella misma y donde las confianzas se generan sólo sobre la base de procesos de mutua apertura. Se podría pensar que en las relaciones puras, que son un fin en sí mismas, el individuo tiene también valor en sí mismo.

En este sentido surge la siguiente pregunta: ¿Es la individuación del individuo, a la vez que un proceso impuesto e inevitable, una posibilidad de resignificar las relaciones de intimidad?

\section{La intimidad de las relaciones interpersonales: valoración del individuo}

Dentro del proceso de individuación del individuo, se puede hipotetizar que el valor en sí mismo se ha ido desgastando, pues son estos sujetos los que deben preocuparse de construirse -considerando todas las variables que la libertad les otorgapara poder relacionarse con los sistemas y estar incluidos en la sociedad.

Esta inclusión no deja de tener un costo, pues el valor del "ser" del individuo no se presenta como uno de los elementos de distinción desde la teoría de sistemas, más bien se introducen a ella a través 
de la opción de comunicaciones específicas que, en ocasiones, pueden anularles el valor del individuo. Sin embargo, este proceso desarrollado en las sociedades modernas trae consigo otra consecuencia, que tiene relación con la valoración de las cualidades y habilidades de los individuos. Esta resignificación del individuo se fortalece en espacios más privativos, es decir, en el espacio de la intimidad de las relaciones intrahumanas. En síntesis, es posible afirmar que las sociedades modernas "se caracterizan por una doble acumulación: un mayor número de posibilidades de establecer relaciones impersonales y una intensificación de las relaciones personales" (Luhmann 1985: 13). Esta doble acumulación se logra entender desde la complejidad de la sociedad moderna, donde las relaciones impersonales permiten que los diversos sistemas se acoplen según su funcionalidad, lo que potencia que se regulen de mejor forma las interdependencias que se producen en las distintas relaciones sociales. Es así que, en el contexto de la sociedad moderna, no se puede pensar en una masificación de las relaciones personales, más bien se puede identificar la intensificación de dichos espacios; "una extensión numérica de este tipo de relaciones tropezaría en cada individuo concreto con un límite insalvable, tras el cual las exigencias que presentarían las relaciones personales tan extensas serían de todo punto imposible de satisfacer. En las relaciones sociales el impulso personal no puede extenderse, sino que ha de intensificarse" (Luhmann 1985:14).

Pese a ello, se resignifica un espacio de valoración distinto: el espacio de la intimidad de las relaciones interpersonales. Es aquí donde el individuo puede sentirse valorado por ser él mismo. En otras palabras, estas relaciones personales, donde se destacan ciertas cualidades o más bien todas las cualidades del individuo, se designarán como "interpenetración intrahumana", es decir “relaciones íntimas”(Luhman, 1985).

En el proceso de la transformación social-de la sociedad estratificada a la funcional- se aprecia que una de las diferenciaciones más potentes ha sido la del sistema personal con el sistema social, puesto que desde la lógica funcional, el individuo debe considerarse como un ser inestable socialmente, sin un lugar fijo. Es por ello que se entiende que el sujeto esté constantemente diferenciándose de su ambiente, lo cual se aprecia en la forma que se diferencia de los demás a través de sus actos y comportamientos. Pese a todas 
las discrepancias posibles entre la individualización provocada y la necesidad de un mundo íntimo y cercano, se ha desarrollado un medio de comunicación simbólico que utiliza el campo semántico de la amistad y del amor (Luhman, 1985)

Luhmann (1985) entiende a los medios de comunicación simbólicamente generalizados, como instituciones semánticas que hacen posible que las comunicaciones aparentemente improbables, puedan realizarse con éxito, pese a ese escaso índice de posibilidad. En este sentido, puede decirse que el amor-como medio simbólico"no es en sí mismo un sentimiento, sino un código de comunicación de acuerdo con cuyas reglas se expresan, se forman o se simulan determinados sentimientos; o se supeditan a dichas reglas o las niegan, para poder adaptarse a las circunstancias que se presenten en el momento en que deba realizarse la correspondiente comunicación" (Luhmann 1895:21). Así, para el medio de comunicación amor, el problema radica en que posee un carácter altamente personal².

El amor se relaciona a los espacios constituidos por las relaciones "amorosas" de las parejas, los espacios íntimos de la familia, las relaciones de amistad, entre otras. Lo relevante en estos espacios es que cada individuo se siente valorado en sí mismo por otro, sin necesidad de exigirse o más bien, sobre exigirse para lograr estar siendo incorporado y acoplado al resto de los sistemas. Los sistemas son autopoiéticos, por lo tanto, los espacios de relaciones interpersonales pueden ser analizados desde estas lógicas, para comprender cómo el individuo se relaciona y se constituye como individuo en ellos.

La transformación de las sociedades de estratificadas a modernas, ha implicado cambios en todas las esferas que las constituyen. Es así que podemos evidenciar estos cambios en instituciones tradicionales como la familia. La diversidad e informalidad en las formas de organizar los vínculos familiares es vista, cada vez más, como un hecho normal. Esta creciente legitimidad se debe, por una parte, a que la cultura de la individualización deja, a las propias personas, decidir sobre la forma de organizar sus vínculos sociales y el derecho de modificarlos (PNUD, 2002).

2 La definición "altamente personal" se usa para expresar todo tipo de comunicación en la cual, el que habla, busca diferenciarse de los otros individuos, es así que el propio sujeto puede convertirse en tema de la comunicación, es decir, que hable de sí mismo. 
Hoy en día existen formas distintas de hacer y ser familia, sin embargo los individuos siguen encontrando en este espacio la posibilidad de conformarse y validarse como tales, la forma de la familia parece tornarse cada día más opcional y flexible. De todas maneras, para la inmensa mayoría de individuos, sus relaciones familiares son lo más importante para la definición de sus identidades personales y para la realización de sus proyectos vitales (PNUD, 2002). Lo anterior no busca obviar que las familias se presentan sin problemas para el desarrollo de las relaciones interpersonales, puesto que éstas también poseen las dificultades que la propia sociedad moderna posee, lo cual influye al desarrollo social de sus integrantes. Si bien no se niega que existan dichas problemáticas, son los mismos individuos las que las relevan como un espacio en que no hay demanda de un sistema mayor, más bien es un espacio que les posibilita la valoración en sí mismos.

Otro de los espacios que cobra una relevancia para la valoración en sí del individuo es aquel que se construye por los lazos de amistad. Por amistad se entiende "el vínculo libremente elegido entre personas sin otra relación previa, que está revestido de los afectos propios de la intimidad: amor, gratuidad, protección, lealtad" (PNUD 2002:228). Estas relaciones implican un desafío para los propios individuos, puesto que -como lo señala Luhman- deben ser ellos mismos los que se transforman en comunicaciones, puesto que deben hablar de sí mismos. Este hablar de la propia individualidad se entiende desde la lógica que ego se debe convertir en una comunicación atractiva para alter, puesto que él será quién tendrá la posibilidad de aceptar/rechazar esta comunicación de ego. Es por ello que se puede afirmar que la amistad no "surge de la nada. Requiere de una imagen positiva y afectiva del otro" (PNUD 2002:228).

Sin embargo, estas relaciones interpersonales se constituyen en este espacio de intimidad que el individuo construye, en la cual es valorado en sí mismo, lo que le posibilita desplegar la gama de habilidades y cualidades que posee de forma arbitraria. Por tanto, es un espacio en que desarrolla una comunicación, un alter y un ego, sin tener la misión última de acoplarse a un sistema mayor; ello posibilita que dicha comunicación se mantenga sólo en este espacio privado.

Un último espacio de intimidad -que se revisará en el presente artículo -se relaciona con los originados en las relaciones de 
pareja. Dichos espacios, al igual que los anteriores, se han ido complejizando, ya que requieren una disponibilidad particular tanto de ego como de alter. Cuando la individualización se fortalece, los vínculos tienden a personalizarse. Cada relación debe constituir su propio guión a través de sus particulares encuentros, diálogos y compromisos. Allí, las emociones tienen un papel central. Esto hace que hoy, para muchas personas, la relación de pareja cobre una importancia inédita en el repertorio de los vínculos sociales (PNUD, 2002).

A pesar del carácter fuertemente subjetivo y emocional que se le otorga a las relaciones de pareja, no existe una real aspiración a la fusión en el otro. En este sentido preciso, la nueva imagen de pareja no es romántica. Más bien al contrario; esta forma de construir la pareja se justifica, precisamente, porque permite realizar y mantener la propia identidad personal.

Puede sugerirse que lo que está cambiando para muchos es la imagen misma del amor. Éste no puede identificarse ya con una relación institucionalizada -como en el matrimonio- ni tampoco con una fusión de individualidades -como en el romanticismo- o con ambas a la vez. Hoy, el amor parece inseparable del marco que le impone la individualización. El reforzamiento de la autonomía individual, el desarrollo y la satisfacción personal, tanto intelectual como emocional y corporal, han de ser los síntomas de un amor verdadero. El amor actual supone un grado de tensión entre individualidades. En resumen, la afirmación de la individualidad da origen a una forma particular de organizar las relaciones de pareja. Ella importa como relación entre individuos y como espacio de potenciación del aprendizaje y del desarrollo personal (PNUD, 2002).

Constatando que la mirada de la pareja ha cambiado, esta sigue siendo uno de los espacios más valorados por los individuos, puesto que en ella se simboliza la opción constante que hace un alter por un ego, considerando las múltiples opciones que se encuentran en el contexto. Las relaciones de parejas tienen cierto símil a la vinculación que se realiza en las relaciones de amistad, sin embargo, los individuos le otorgan un valor mayor por la decisión que les implica. Escogen por un alter, y esta decisión es la que se va confirmando a través del tiempo. Junto con ello, la relación de pareja otorga un espacio de intimidad que los individuos no encuentran en 
otro tipo de relaciones personales, puesto que es en seno de ellas en que logran desplegarse en un grado distinto al realizado en los otros tipos de relaciones -mencionados en este artículo- logrando construir un sistema propio de los involucrados.

\section{Problemáticas que enfrenta el individuo al construir un espacio de intimidad}

Como se ha visto, los espacios de intimidad que se producen, ya sea en las relaciones de pareja, familia, amigos u otro tipo de relaciones sociales íntimas, otorgan a los individuos mayores posibilidades de ser valorados en sí mismos.

Sin embargo, se presenta la "falta de tiempo" como una amenaza a la mantención de espacios de intimidad, pues las demandas de los sistemas parciales dejan poco tiempo cronológico para desarrollar otros aspectos de la vida, tales como la familia, los amigos, el descanso, entre otros. El tiempo dedicado a la tarea de acoplarse con dichos sistemas, de manera de ser incluido, dificulta tomar distancia de sus exigencias y relativizarlas, impone un ritmo que deja poco tiempo para otras cosas (PNUD, 2002).

Asimismo, las demandas de los otros o las que imponen las relaciones con los otros, refieren a las imposiciones sobre las relaciones con los otros íntimos, en especial la familia, los amigos, la pareja. Lealtad, dedicación, afectos, confianza, sacrificio, son las demandas en este plano (PNUD, 2002); esto generaría un aumento de las exigencias a las que los individuos se ven sometidos tanto en los ámbitos "externos" como "internos". Asimismo el individuo corre riesgo de crear dependencia o mutua dependencia con el otro, provocando relaciones nocivas y limitando la libertad de sus integrantes, aumentando las exigencias a las que se ve sometido y restringiendo la posibilidad de autorrealización en este espacio.

Por otro lado, en una época de lo que Giddens llama "el amor confluente", las relaciones de intimidad perduran lo que la gratificación de uno de los asociados, los lazos son desde el principio sólo "hasta nuevo aviso" y el apego de hoy puede convertirse en la frustración de mañana (Beck, 2003). Es así como se aprecia que, en las sociedades modernas, los espacios de intimidad relacionados con la familia y la pareja se han judicializado crecientemente; 
ejemplo de ello, es la figura legal del divorcio, o la legalización de las responsabilidades parentales que, en ocasiones, son necesarias de definir cuando la pareja decide deshacer su vínculo.

En este sentido los cambios generados en las relaciones familiares, con la pareja y con los amigos, los cambios ocasionados en la vida laboral y en las instituciones que representan las certezas sociales terminan, tarde o temprano, afectando como un todo al orden y al sentido de la existencia previamente establecido (PNUD, 1998).

\section{Conclusiones}

A través de lo expuesto en el presente artículo, se hace posible dar respuesta a la pregunta que originó este ensayo: ¿Es la individuación del individuo, a la vez que un proceso impuesto, una posibilidad de resignificar las relaciones de intimidad?

Si bien la sociedad moderna con la consecuente diferenciación funcional de los sistemas parciales, ha impulsado un tipo de relación impersonal, donde la individuación ha sido el resultado, el individuo ha logrado integrar estas demandas y, a la vez, equilibrarlas con la propia necesidad. Esto implica que si bien es parte del sistema comunicacional que conforma a la sociedad, logra desarrollar y construir espacios de carácter íntimo y/o privado, tales como la pareja, la familia, los amigos, en los cuales consigue ser valorado como entidad en sí misma.

Es así que se nos hace posible afirmar que en la actualidad existe una resignificación de las relaciones interpersonales íntimas, puesto que ya no son instituciones que constituían a las sociedades estratificadas -como el matrimonio- más bien hoy se significan desde la propia constitución del individuo como desde su propias elecciones.

Asimismo, a pesar que estos espacios de intimidad no están exentos de dificultades y/o amenazas, influenciados por procesos de contingencia y doble contingencia, el individuo se encuentra en una constante búsqueda de consolidación de relaciones de intimidad, donde pueda sentirse valorado, autorrealizado y donde pueda confirmar también la vivencia del otro. 


\section{Referencias bibliográficas}

Avaria, A. (2001). Discapacidad: Exclusión/Inclusión. Recuperado el 12 de mayo de 2009. De www.independentliving. org/docs7/saavedra200109.html

Beck, U. y Beck, E. (2003). La Individualización. El Individualismo institucionalizado y sus consecuencias sociales y políticas. Barcelona: Paidós.

Giddens, A. (1995). Modernidad e Identidad del Yo. El yo y la sociedad en la época contemporánea. Barcelona: Península/Ideas.

Giddens, A. (1998). Las consecuencias de la Modernidad. En: Luhmann, N. Complejidad y Modernidad. Madrid: Trotta.

Giddens, A. (2000).Un mundo desbocado. Los efectos de la globalización en nuestras vidas. Madrid: Taurus.

Luhmann, N. (1998). Complejidad y modernidad. Madrid: Trotta.

Luhmann, N. (1985). El amor como pasión. La codificación de la pasión. Madrid: Penínsulas.

Mascareño, A. (2006). Sociología de la Felicidad: lo incomunicable. [Versión electrónica]. Teología y Vida, 47 (2-3), pp. 190-208.

PROGRAMA DE LAS NACIONES UNIDAS PARA EL DESARROLLO (1994). Informe Desarrollo Humano en Chile. El poder: ¿para que y para quien?. Santiago, Chile: AUTOR.

(1998). Elementos para la comprensión de la Seguridad Humana en la modernidad. En Informe Desarrollo Humano en Chile. Las paradojas de la Modernización. Santiago, Chile: AUTOR.

(2002). Informe Desarrollo Humano en Chile. Nosotros los chilenos: un desafío cultural. Santiago, Chile: AUTOR.

Robles, F. (1999). El sujeto y la cotidianidad. Santiago, Chile: Sociedad Hoy. 